\title{
Shroom expression is attenuated in pulmonary arterial hypertension
}

\author{
J. Sevilla-Pérez*, M. Königshoff*, G. Kwapiszewska\#, O.V. Amarie*, W. Seeger*, \\ N. Weissmann*, R.T. Schermuly*, R.E. Morty* and O. Eickelberg*
}

ABSTRACT: Shroom is a PDZ-domain protein involved in the regulation and maintenance of cytoskeletal architecture by binding to actin. Hypertrophy and altered actin organisation of pulmonary arterial smooth muscle cells (PASMC) is a hallmark of pulmonary arterial hypertension (PAH). The aim of the present study was to localise and characterise Shroom expression in the lung in experimental and idiopathic PAH (IPAH).

Shroom expression and localisation in hypoxia-induced PAH in mice and IPAH in humans, in vivo, as well as in primary PASMC, in vitro, was assessed by quantitative RT-PCR, immunofluorescence, laser-assisted microdissection and immunohistochemistry.

Shroom localised exclusively to PASMC (both bronchial and vascular) in mouse and human lungs. Both in vivo and in primary PASMC, in vitro, Shroom exhibited spatially similar expression with $\alpha$-smooth muscle actin ( $\alpha$-SMA). Shroom expression was significantly reduced in the mouse model of PAH, in primary murine PASMC exposed to hypoxia, and in primary PASMC isolated from patients with IPAH. The ratio between Shroom and $\alpha$-SMA RNA expression further confirmed Shroom downregulation in both mouse and human PASMC.

In summary, Shroom localises exclusively to pulmonary smooth muscle cells. Shroom downregulation in pulmonary arterial hypertension suggests a link between Shroom expression and pulmonary arterial smooth muscle cell hypertrophy in pulmonary arterial hypertension.

KEYWORDS: Actin-binding proteins, animal model, hypoxia, pulmonary arterial smooth muscle cells

$\mathbf{T}$ he normal pulmonary circulation represents a low-pressure, high-flow vascular system. Pulmonary arteries are characterised by a delicate tunica intima and media, which can be remodelled under disease conditions. Vascular remodelling, along with sustained vasoconstriction, leads to a decrease in the luminal area and, if those changes persist chronically, to the development of pulmonary arterial hypertension (PAH) $[1,2]$. $\mathrm{PAH}$ is a devastating disorder characterised by sustained elevation of pulmonary arterial pressures, obstruction of small pulmonary arteries by plexiform and concentric lesions, intimal fibrosis, and medial hypertrophy [3, 4]. Abundant evidence from clinical and genetic studies suggest the involvement of the serotonin, bone morphogenetic protein and angiopoietin systems in disease pathogenesis [4, 5]. The severity of $\mathrm{PAH}$ is determined, at least in part, by the degree of structural changes that occur within the wall of pulmonary arteries. Notable amongst these structural changes are extracellular matrix deposition, cell proliferation and hypertrophy of pulmonary artery smooth muscle cells (PASMC), which represent key processes that lead to medial and/or adventitial thickening of pulmonary arteries $[4,6]$. The development of PAH is therefore closely linked to enhanced PASMC activation and proliferation, which is preceded by reorganisation of the actin-myosin cytoskeleton. Activated PASMCs display reduced contractility but enhanced motility and growth [7] Importantly, stimuli that play an essential role in $\mathrm{PAH}$ pathogenesis, such as serotonin, have been shown to induce reorganisation of the actin cytoskeleton [8].

The cytoskeleton is an integrated, dynamic network of three major types of protein filaments, actin fibres, intermediate filaments and microtubules, which regulate cell motility, intracellular transport and PASMC contraction [9]. Three actin isoforms exist in higher eukaryotes: $\alpha$-actin, which is largely present in muscle cells, and $\beta$ - and $\gamma$ actin, which are also abundant in non-muscle cells. The actin pools are organised and regulated by a large number of specialised actin-binding proteins [10]. These actin-binding proteins are
AFFILIATIONS

*Depts of Medicine, and

\#Pathology, University of Giessen Lung Centre, Justus Liebig University Giessen, Giessen, Germany.

CORRESPONDENCE

0 . Eickelberg

University of Giessen Lung Centre

Dept of Medicine ॥

Aulweg 123

Room 6-11

D-35392 Giessen

Fax: 496419942309

E-mail: oliver.eickelberg@

innere.med.uni-giessen.de

Received:

April 132007

Accepted after revision:

May 022008

STATEMENT OF INTEREST

None declared. 
responsible for the stability, dynamics, higher order organisation and subcellular distribution of the actin cytoskeleton [11]. Members of the recently described Shroom family of proteins represent one group of such actin-binding proteins [12].

Shroom family members possess an N-terminal PDZ domain necessary for protein-protein interaction, an intermediate Apx/Shrm domain (ASD)1 required for actin binding and the C-terminally positioned ASD2, necessary for actomyosin contraction [12]. Shroom participates in vertebrate development by regulating the recruitment and maintenance of the actomyosin network and determines cell morphology in nonmuscle cells, as initially observed in cell culture experiments $[12,13]$. Furthermore, Shroom is essential for neural-tube closure in both mice and frogs [12]. In the mouse, the shrm gene (encoding Shroom), positioned on chromosome 5, encodes two transcripts that give rise to two different putative protein products. These transcripts have been designated according to their size, shrmL (1,986 amino acids) and shrmS (1,808 amino acids) [12].

The aim of the present study was to investigate Shroom expression in the lung, following the hypothesis that expression levels of this actin-binding protein might be altered during the development of PAH. The expression and specific localisation of Shroom was analysed in an experimental model of PAH (hypoxia-induced PAH in mice), as well as in human idiopathic PAH (IPAH) samples. Shroom expression was downregulated in lung homogenates obtained from experimental animals with hypoxia-induced PAH. Importantly, Shroom was selectively expressed by PASMCs in mice and humans. Shroom expression was significantly downregulated in primary murine PASMCs cultured under hypoxic conditions in vitro, as well as in PASMCs cultured from the lungs of patients with IPAH compared with PASMCs cultured from healthy human lungs. The present authors propose that Shroom may play an essential role in the homeostasis of the PASMC actin cytoskeleton. Since its expression is attenuated in PASMCs in PAH, restoration of normal levels and functions of actin-binding proteins, such as Shroom, in PAH may serve as an attractive option to modulate the severely altered PASMC function in this disease.

\section{MATERIALS AND METHODS}

\section{Animal procedures}

Adult male C57Bl/6J mice (20-24 g) were obtained from Charles River WIGA GmbH (Sulzfeld, Germany). Animals were housed under controlled temperature $\left(22^{\circ} \mathrm{C}\right)$ and lighting (12/12-h light/dark cycle), and were allowed food and water ad libitum. All animal experiments were performed in accordance with institutional and national guidelines for the care and use of experimental animals. For hypoxic exposures, male $\mathrm{C} 57 \mathrm{Bl} / 6 \mathrm{~J}$ mice were placed in a ventilated chamber system with an inspiratory oxygen fraction of 0.10 . After 2, 7 or 21 days, mice were sacrificed by intraperitoneal injection of sodium pentobarbital, and lungs were extracted (six to seven animals per condition). The lungs were either immediately snap-frozen in liquid nitrogen or harvested and processed for histological embedding and sectioning. Haemodynamic studies were performed in all study animals, as previously reported [14-16].

\section{Human tissues}

Lung tissue biopsies were obtained from seven patients with IPAH (four female, three male; mean \pm SEM age $34.5 \pm 10.5 \mathrm{yrs}$ ) and six control subjects (organ donors; three female, three male; $37.8 \pm 14.1 \mathrm{yrs}$ ). None of the IPAH patients exhibited mutations in the bone morphogenetic protein receptor, type 2 gene, BMPR2. Samples were either fixed in $4 \%$ (weight/volume) paraformaldehyde or snap-frozen in liquid nitrogen within $30 \mathrm{~min}$ of explantation. All investigations involving human tissues were performed in accordance with the Declaration of Helsinki. The study protocol was approved by the Ethics Committee of the Justus-Liebig University School of Medicine (approval number AZ 31/93; Giessen, Germany). Informed consent was obtained from each subject or next-of-kin.

\section{Laser-assisted microdissection}

Microdissection was performed as described previously [17]. Briefly, cryo-sections from lung tissue were mounted on glass slides. After hemalaun staining, the sections were immersed in 70, 96 and 100\% ethanol. Intrapulmonary arteries with a diameter of $250-500 \mu \mathrm{m}$ were selected and microdissected under optical control using the Laser Microbeam System (Carl Zeiss Microimaging Gmbh, Bernried, Germany). Vessel profiles were isolated with a sterile $30-G$ needle and transferred into a reaction tube containing $200 \mu \mathrm{L}$ RNA lysis buffer [17].

\section{Cell culture}

Mouse PASMCs were isolated from pulmonary arteries using the explant method, as described previously [14], and cultured in Dulbecco's modified Eagle medium-F12 supplemented with 10\% foetal calf serum (PAA Laboratories, Pasching, Austria). Only passages 3-6 were used for the present experiments, during which PASMCs routinely exhibited typical spindleshaped morphology and stained positive for $\alpha$-smooth muscle actin ( $\alpha$-SMA; Sigma-Aldrich, St Louis, MO, USA). For hypoxia exposures, cells were placed in a chamber simulating hypoxic conditions with a water-saturated gas mixture of $1 \% \mathrm{O}_{2}, 5 \%$ $\mathrm{CO}_{2}$ and $94 \% \mathrm{~N}_{2}$ at $37^{\circ} \mathrm{C}$ for 24 or $48 \mathrm{~h}$.

\section{RNA extraction}

RNA was extracted from lung homogenates, microdissected arteries or PASMCs following the RNeasy Mini protocol (Qiagen, Hilden, Germany). Total RNA concentration was determined spectrophotometrically at $260 \mathrm{~nm}$.

\section{RT-PCR}

RNA isolated from lung homogenates or PASMCs was reverse-transcribed using Moloney murine leukemia viral reverse transcriptase and Oligo $(\mathrm{dT})_{15}$ primers (Promega, Madison, WI, USA). Semi-quantitative RT-PCR was performed using GoTaq Flexi DNA Polymerase (Promega) in a total volume of $25 \mu \mathrm{L}$. The PCR products were resolved by agarose gel electrophoresis and visualised by ethidium bromide staining. Quantitative real-time RT-PCR was performed in a 7500 Fast Real-Time PCR System (Applied Biosystems, Foster City, CA, USA). All reactions (in a final volume $25 \mu \mathrm{L}$ ) were carried out using Platinum ${ }_{\circledR}$ SYBR $_{\circledR}$ Green qPCR SuperMixUDG (Invitrogen, Carlsbad, CA, USA). The cycling conditions were as follows: $95^{\circ} \mathrm{C}$ for $6 \mathrm{~min}$, followed by 45 cycles of $95^{\circ} \mathrm{C}$ for $5 \mathrm{~s}, 60^{\circ} \mathrm{C}$ for $5 \mathrm{~s}$ and $72^{\circ} \mathrm{C}$ for $30 \mathrm{~s}$. Specific amplification of PCR products was confirmed by melting-curve analysis and 
gel electrophoresis. Relative changes in gene expression were analysed using equation 1 and 2 or 3 :

$$
\begin{gathered}
\Delta \Delta \mathrm{Ct}=\Delta \text { Cthypoxia }-\Delta \text { Ctnormoxia } \\
\Delta \mathrm{Ct}=\mathrm{Ct} \text { shrm }-\mathrm{Ct} \text { acta } 2 \\
\Delta \mathrm{Ct}=\text { Ctreference gene }-\mathrm{Ct} \text { shrm }
\end{gathered}
$$

where $\mathrm{Ct}$ is the cycle number where the fluorescence value is above the determined threshold and $\Delta$ is change. The intronspanning primers used for semi-quantitative and quantitative RT-PCR are indicated in table 1 . The porphobilinogen deaminase $(p b g d)$, hypoxanthine-guanine phosphoribosyltransferase (hprt) or glyceraldehyde 3-phosphate dehydrogenase (gapdh), ubiquitously as well as consistently expressed genes, which are free of pseudogenes, were used as reference genes [18].

\section{Immunostaining}

Protein localisation was assessed using paraffin-embedded tissue sections and antibodies specifically recognising Shroom (sc-10310 (E-19); Santa Cruz Biotechnology, Santa Cruz, CA, USA; at a dilution of 1:100) or $\alpha$-SMA (A5228; Sigma-Aldrich; at a dilution of 1:1000). Paraffin sections $(3 \mu \mathrm{m})$ were mounted on poly-L-lysine-coated slides, dewaxed and rehydrated by immersion in ethanol (70, 95 or 100\%) and PBS (PAA Laboratories). After antigen retrieval, endogenous peroxidase activity was blocked by incubation with $3 \% \mathrm{H}_{2} \mathrm{O}_{2}$ for $20 \mathrm{~min}$. Antigens were detected according to the manufacturer's instructions (Histostain Plus Kit; Invitrogen) [18].

For single cell analysis, PASMC were seeded in eight-well chamber slides, fixed and permeabilised with methanol. Incubation with primary antibodies was performed for $1 \mathrm{~h}$ at room temperature, followed by incubation with fluorescein isothiocyanate-conjugated rabbit anti-goat (at a dilution of 1:300; Invitrogen) or Texas Red-conjugated goat anti-mouse (at a dilution of 1:400; Invitrogen) antibodies, for Shroom and $\alpha$-SMA, respectively. Cell nuclei were labelled with 4'-6-diamidino-2phenylindole (Roche Diagnostics, Mannheim, Germany). Cells were visualised by fluorescent microscopy using an AS-MDW microscope (Leica, Bensheim, Germany) [14].

\section{Statistical analysis}

Values are presented as mean \pm SEM. The means of indicated groups were compared using a two-tailed unpaired t-test, or a one-way ANOVA with Tukey's honestly significantly different post hoc test for studies with more than two groups. A p-value $<0.05$ was considered statistically significant.

\section{RESULTS}

\section{Shroom expression and localisation in hypoxia-induced PAH}

Shroom expression was initially assessed in the mouse model of chronic hypoxia-induced $\mathrm{PAH}$, using lung homogenates from mice maintained under normoxic (21\% oxygen) or hypoxic (10\% oxygen) conditions for 1, 7 or 21 days. Levels of mRNA encoding Shroom were decreased by up to $50 \%$ in the lungs of mice exposed to chronic hypoxia, as assessed by semi-quantitative RT-PCR (fig. 1a) and corresponding densitometric analysis (fig. 1b). Additionally, in order to accurately quantify the RNA expression levels of shrm and smooth muscle alpha-actin (acta2) in the lung, quantitative real-time RT-PCR was performed. As a positive control, expression levels of phosphoglycerate kinase ( $p g k$ ), which has been described to be upregulated by hypoxia [19], were first assessed. Indeed, $p g k$ mRNA levels were elevated in mice that developed elevated pulmonary artery pressures and vascular remodelling in response to hypoxia (fig. 2a). In contrast, shrm expression was downregulated in the lungs of those mice after a 21-day exposure to hypoxia (fig. 2a); acta2 levels remained unchanged (fig. 2a). Normalisation of shrm expression levels for acta2, which would demonstrate changes in shrm expression in the PASMC fraction of the lung homogenates, further confirmed that shrm expression was downregulated in the lungs of mice after a 1- and 21-day exposure to hypoxia (fig. 2b).

In order to localise Shroom protein in the murine lung, immunohistochemical analyses were undertaken; these demonstrated that Shroom localised exclusively to PASMCs, surrounding both the bronchial and vascular structures (fig. 3). Immunohistochemical staining of adjacent sections further showed that Shroom expression occurred in a spatially similar pattern with $\alpha$-SMA staining, supporting its predominant

TABLE 1 Sequences of forward and reverse primers used for semi-quantitative and quantitative real-time RT-PCR

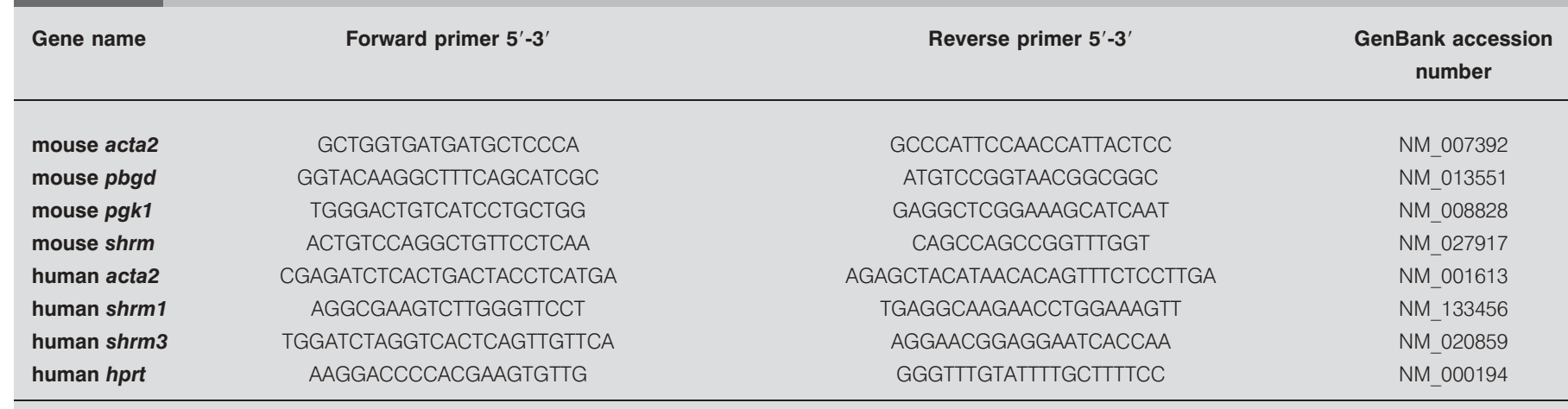

acta: smooth muscle alpha-actin; pbgd: porphobilinogen deaminase; pgk: phosphoglycerate kinase; shrm: Shroom; hprt: hypoxanthine-guanine phosphoribosyltransferase. 
a)
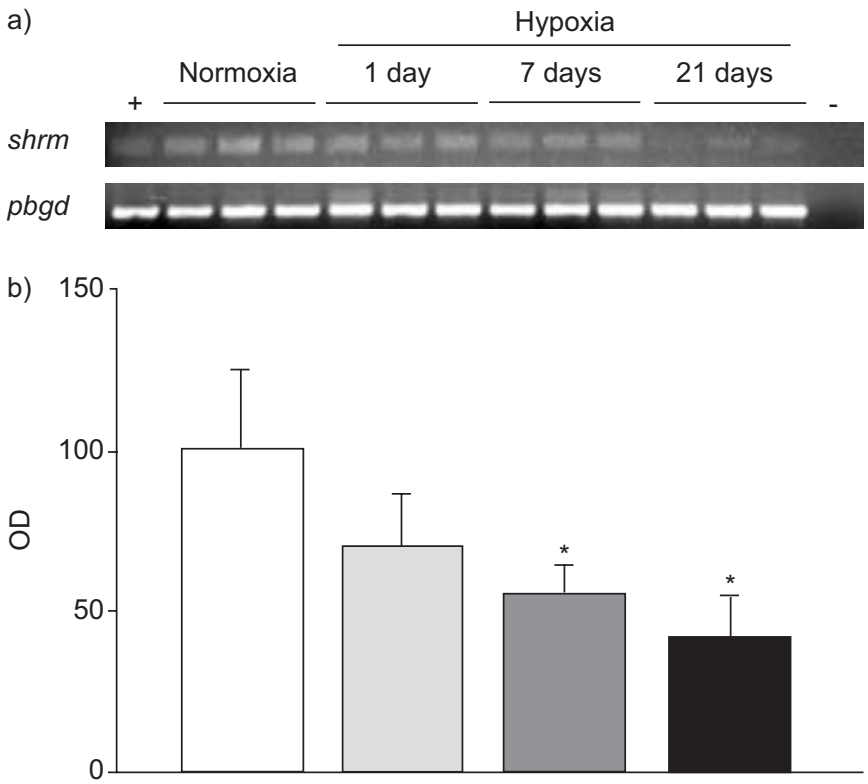

FIGURE 1. Shroom mRNA expression in mouse lung homogenates. a) The shrm gene was expressed in lungs from mice maintained under normoxic conditions. The shrm expression was downregulated in the lungs of mice exposed to chronic hypoxia for up to 3 weeks, as assessed by semi-quantitative RT-PCR. The porphobilingen (pbgd) gene was used as internal loading control. Positive control samples (+) contained commercial total RNA, negative control samples (-) omitted reverse transcriptase. b) Histogram representing densitometric analysis of the gene expression ratio shrm/pbgd. $\square$ : normoxia; $\square$ : hypoxia for 1 day; $\square$ : hypoxia for 7 days; : hypoxia for 21 days. Data are presented as mean \pm SEM of three mice per condition and are representative of the same trends observed with at least six other mice per condition. OD: optical density of the amplicon assessed by densitometry. ${ }^{*}: \mathrm{p}<0.05$.

expression in PASMCs (fig. 3). These observations validate the present approach to normalise shrm for acta2 gene expression, in order to explore changes in shrm expression in the smooth muscle fractions of lung homogenates. When comparing sections from mice subjected to chronic hypoxia with those from normoxic animals, an increase in $\alpha$-SMA staining was observed, as expected, but a decrease in Shroom staining in the PASMC was also observed (fig. 3), which is consistent with the present data generated by semi-quantitative and quantitative RT-PCR analyses of whole lung homogenates (figs 1 and 2).

Next, laser-assisted microdissection was performed in order to specifically isolate RNA from intrapulmonary arteries with a diameter of $250-500 \mu \mathrm{m}$ from mice maintained under normoxic or hypoxic conditions, since PASMCs were the cell type with the highest shrm expression in vivo (fig. 4a). The shrm expression levels were generally stable in these samples (although a small but significant increase was observed after a 21-day hypoxia exposure), as assessed by quantitative RTPCR (fig. 4b). In contrast, acta2 mRNA expression was significantly elevated after 21 days of hypoxia (fig. $4 \mathrm{~b}$ ). As such, normalisation of shrm mRNA expression for acta2 mRNA expression clearly demonstrated a pronounced downregulation of shrm in the smooth muscle layer of intrapulmonary arteries (fig. 4c).
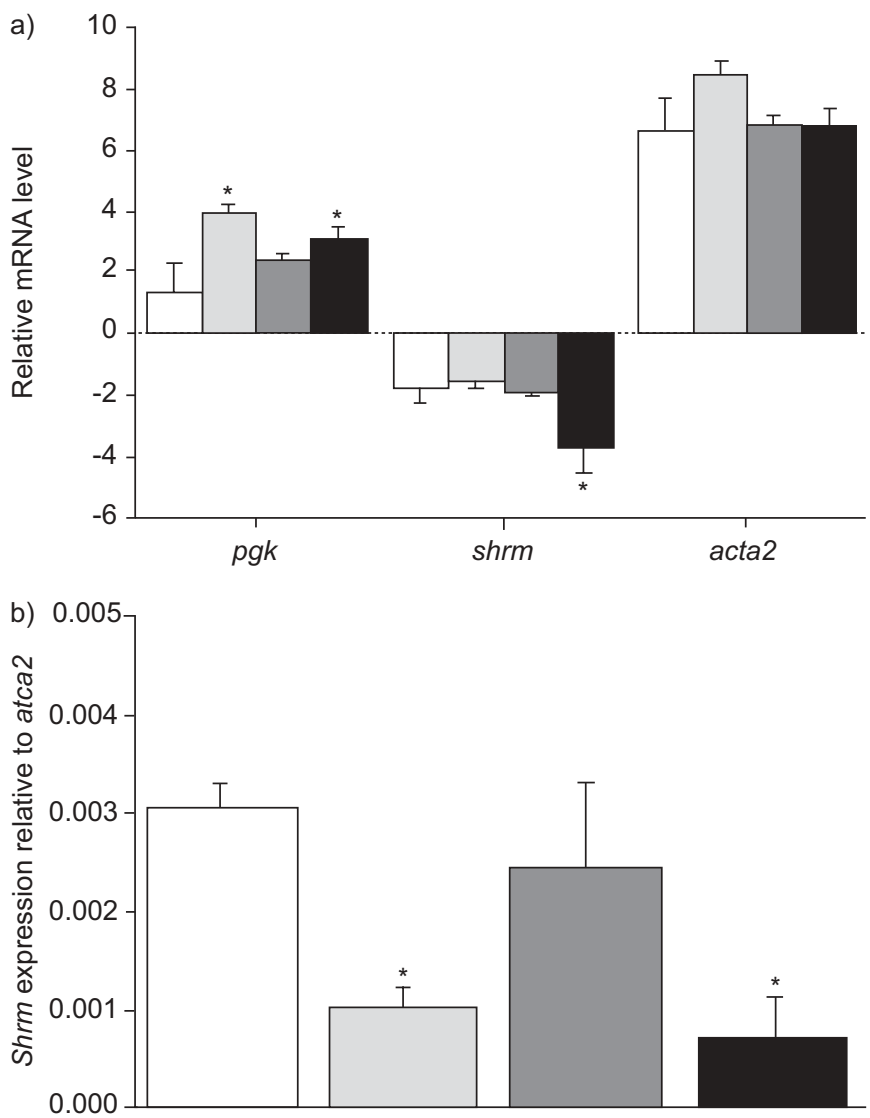

FIGURE 2. Quantitative real-time RT-PCR of shrm gene expression in mouse lung homogenates. a) The mRNA levels of phosphoglycerate kinase (pgk), shrm or $\alpha$-smooth muscle cell actin (acta2) were assessed in the lungs of mice exposed to

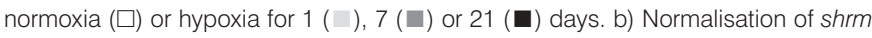
for acta2 gene expression, obtained from the data presented in a). Data are presented as mean \pm SEM obtained from three mice per condition and are representative of the same trends observed with at least six other mice per condition. *: $p<0.05$

\section{Shroom expression and regulation by hypoxia in primary PASMC}

Levels of shrm mRNA expression in PASMC maintained under normoxic or hypoxic conditions were then determined. The shrm expression was significantly downregulated under low oxygen conditions, as assessed by semi-quantitative (fig. 5a) and quantitative (fig. 5b) RT-PCR, using RNA isolated from PASMC maintained under normoxic (21\% oxygen) or hypoxic ( $1 \%$ oxygen) conditions for 24 or $48 \mathrm{~h}$ (fig. $5 \mathrm{a}$ and b). The $p g k$ expression levels again served as a positive control for hypoxia-induced effects (fig. 5b) [19].

Given the apparent regulation of shrm expression by hypoxia, the promoter and enhancer regions of the murine shrm gene were screened for the presence of putative hypoxia-inducible response elements (HREs) at a distance of 5,000 bp upstream and downstream of the coding region. These HRE are of particular interest, as they indicate regulation of mRNA expression by hypoxia-inducible factor (HIF)-1 [19]. An automated computational analysis using the gene prediction method was employed to detect the consensus HREs 

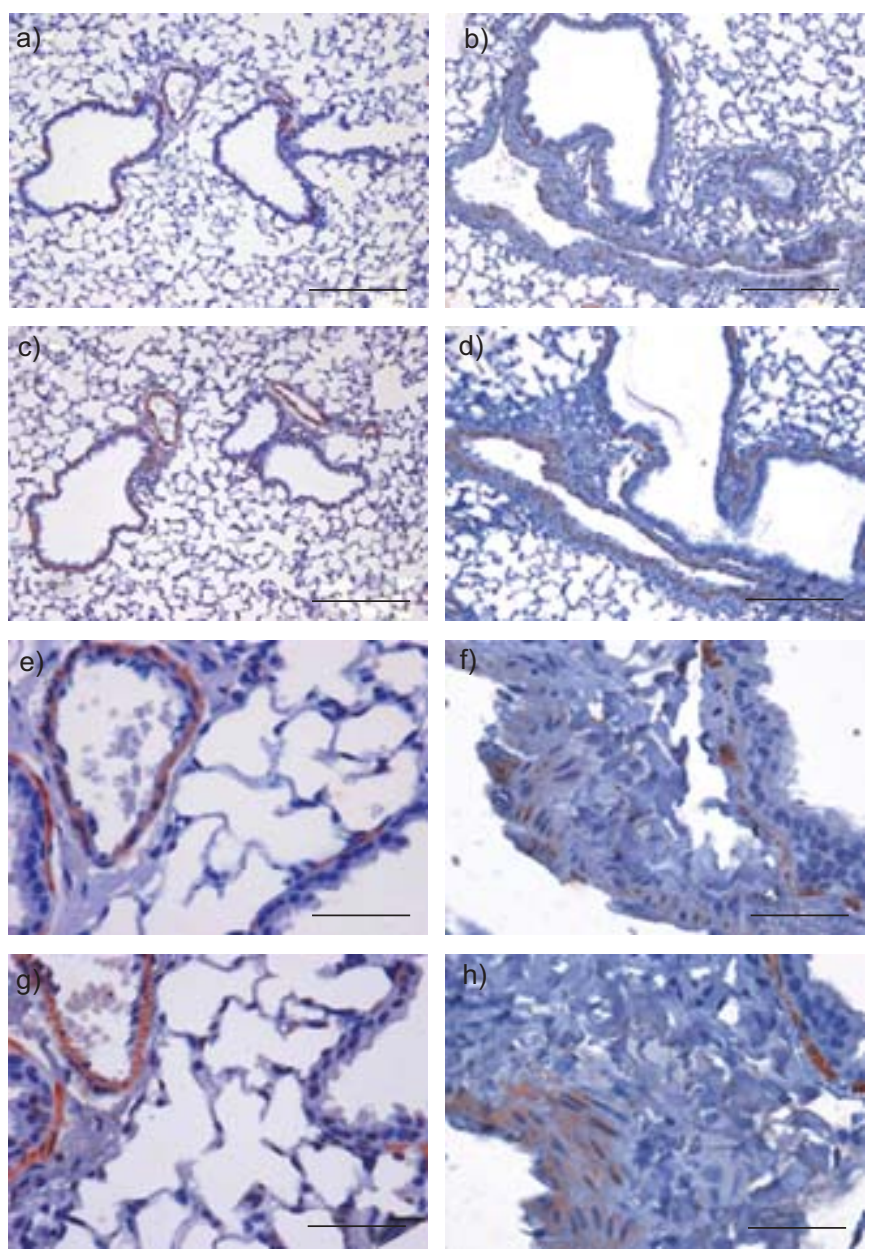

FIGURE 3. Shroom protein localisation in the mouse lung. Representative serial immunohistochemical sections illustrate a similar spatial expression of Shroom (a, b, e and f) and $\alpha$-smooth muscle cell actin (c, d, g and h) in smoothmuscle cells, both in low (a- d) and high (e-h) magnification. a, c, e, g) Staining of normoxic mouse lungs (21\% oxygen, 3 weeks) and $b, d, f, h$ ) staining of hypoxic mouse lungs (10\% oxygen, 3 weeks). Images are representative for three mice per condition. a-d) Scale bars $=200 \mu \mathrm{m}$, e-h) scale bars $=50 \mu \mathrm{m}$.

(ACGTGS, with $\mathrm{S}$ being either $\mathrm{G}$ or C) [20]. Interestingly, four HREs were detected in the shrm promoter and enhancer, located at positions -1573 and +1792 of the sense strand and at -1650 and +641 of the antisense strand (fig. 5c), indicating that HIF-1 may indeed be involved in negative regulation of shrm expression under hypoxic conditions.

Immunofluorescence analysis of murine primary PASMCs maintained under normoxic conditions illustrated a cytoplasmic localisation pattern for Shroom and, using double immunofluorescence, confirmed its co-localisation with $\alpha$ SMA (fig. 6).

\section{Shroom expression and localisation in human lungs}

Shroom localisation was then assessed in control human lungs from healthy transplant donors or in lungs obtained from IPAH patients (none of which exhibited exonic BMPR2 mutations). In the human lung, Shroom protein staining was
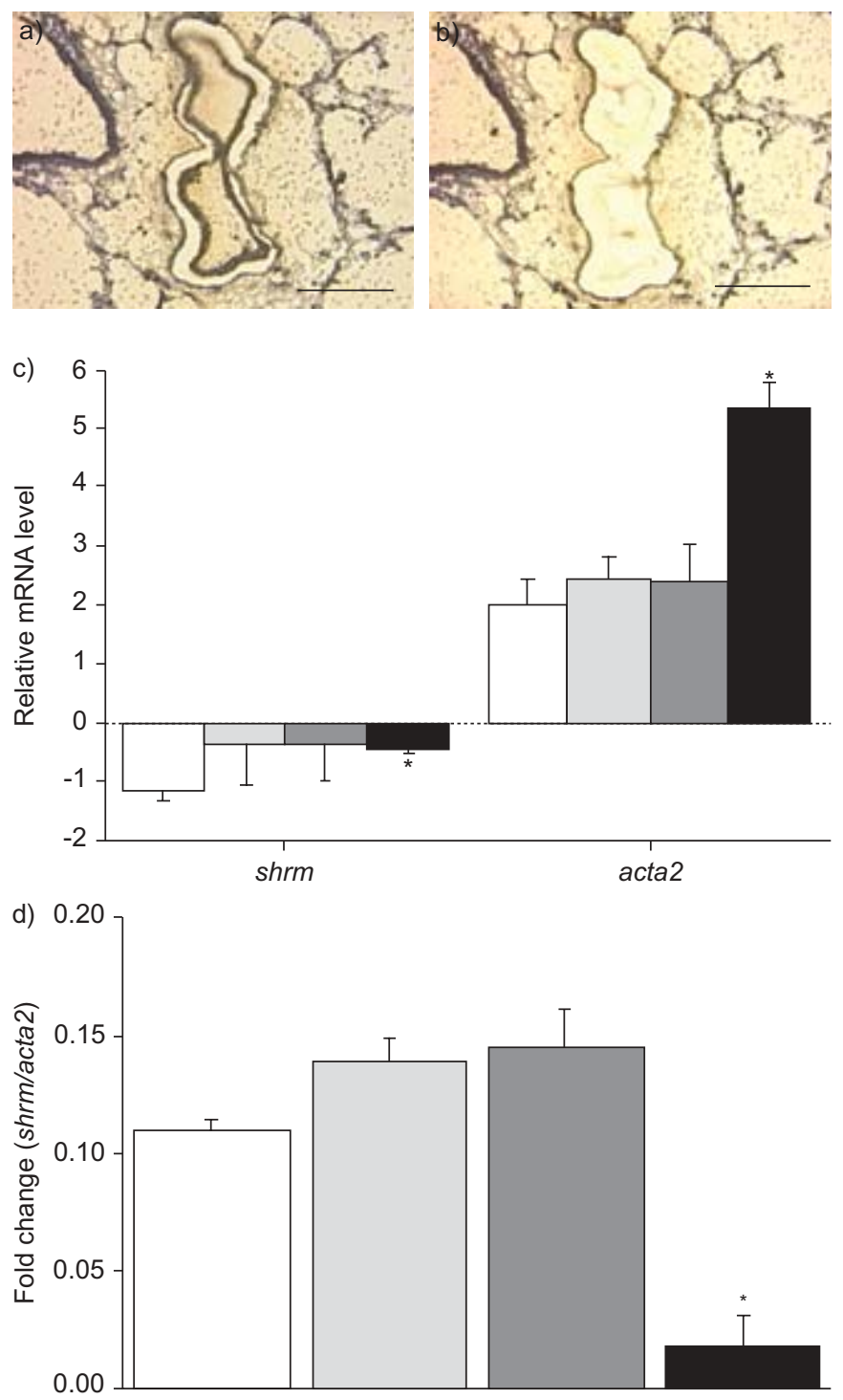

FIGURE 4. Shroom expression in microdissected intrapulmonary arteries. a and b) Representative images showing laser-assisted microdissection of small arteries. c) The shrm and $\alpha$-smooth muscle cell actin (acta2) mRNA gene expression levels in microdissected intrapulmonary arteries from mice maintained under normoxic $(\square)$ or hypoxic conditions for $1(\square), 7(\square)$ and 21 ( $\square$ ) days is represented by $\Delta \mathrm{Ct}$ values (change in the cycle number where the fluorescence value is above the determined threshold). d) Normalisation of shrm for acta2 gene expression, calculated from the data presented in b). Data are presented as mean \pm SEM. *: $p<0.05$

observed predominantly in PASMCs, with a lower intensity in bronchial PASMC (fig. 7), suggesting a similar staining pattern to mouse lungs (compare figs 7 and 3). Staining was most intense within the tunica media of pulmonary arteries. Furthermore, Shroom exhibited a spatially similar expression pattern with $\alpha$-SMA, supporting its predominant expression in PASMC (fig. 7). Shroom protein was also detected in PASMCs of remodelled pulmonary arteries in IPAH samples but only weakly expressed in PASMC within plexiform lesions (fig. 7e and $f$, respectively). 
a)

$\frac{24 \mathrm{~h}}{\frac{\text { Hormoxia }}{2} \stackrel{\text { Hypoxia }}{\text { Normoxia }} \text { Hypoxia }}$

pbgd

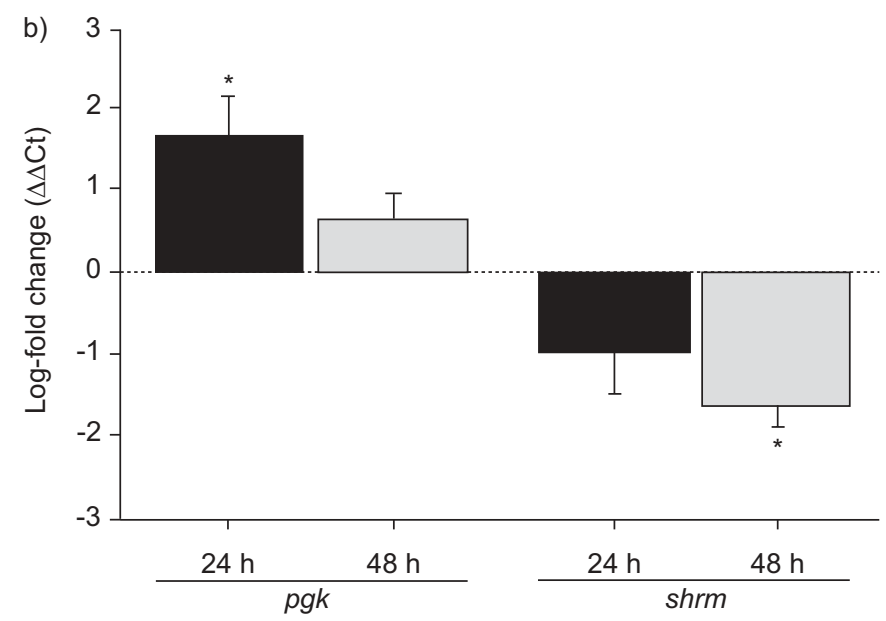

c)

$\underset{-1650}{-1573} \square / C_{+641}^{+1792}$

FIGURE 5. Shroom expression and regulation in mouse primary pulmonary arterial smooth muscle cells (PASMCs). The shrm mRNA expression was assessed by a) semi-quantitative and b) quantitative RT-PCR. Attenuation of shrm expression was observed in PASMCs cultured under hypoxic conditions (1\% oxygen) for 24 and $48 \mathrm{~h}$. The porphobilingen (pbgd) expression was used as internal loading control, while phosphoglycerate (pgk) gene expression served as positive control for hypoxic conditions. c) Schematic illustration of the hypoxia-responsive elements in the shrm promoter and enhancer regions. $\Delta \Delta \mathrm{Ct}$ : log-fold change in gene expression between hypoxia and normoxia, as defined in detail in Materials and Methods: RT-PCR. *: $p<0.05$.
Levels of mRNA encoding shrm1 and shrm3, as well as acta2 were then assessed in homogenates obtained from healthy human lungs or lungs from IPAH patients (fig. 8). Both shrm1 and acta2 mRNA levels were upregulated in lung homogenates of IPAH patients compared with homogenates from healthy control lungs, while shrm3 levels remained unchanged (fig. 8a). Normalisation of shrm1 for acta2 mRNA levels suggested that PASMC-specific changes in shrm1 expression were not evident when comparing lung homogenates from IPAH patients with healthy donor controls (fig. 8b). When PASMC were cultured from lungs of healthy donors or patients with IPAH, no absolute changes in shrm1 mRNA expression were observed but a pronounced increase in acta2 mRNA expression was observed (fig. 9a). Normalisation of shrm1 for acta2 mRNA levels in cultured PASMC, however, clearly demonstrated a downregulation of shrm1 expression in PASMC from IPAH patients versus healthy donors (fig. 9b).

\section{DISCUSSION}

The novel observations reported within the present study are as follows. 1) For the first time, the presence of the actinbinding protein Shroom in mouse and human lungs was demonstrated. 2) Shroom was demonstrated to be a smooth muscle-specific protein in mouse and human lungs and its expression was attenuated during the development of experimental PAH in the mouse. 3) Shroom was localised to the cytoplasm, along with $\alpha$-SMA, in mouse PASMCs. 4) While $\alpha$ SMA expression increased in the disease state, Shroom expression relative to $\alpha$-SMA was maintained or downregulated in PASMCs in both the human disease and the mouse model of hypoxia-induced PAH.

In order to begin to decipher the role of Shroom in the lung vasculature, a mouse model of PAH was employed, where elevated pulmonary artery pressures and vascular remodelling were induced by chronic exposure to normobaric hypoxia. In this model, analysis of RNA derived from lung homogenates revealed a decreased expression of shrm, suggesting a potential role during disease development. Moreover, Shroom protein staining was less pronounced in PAH-induced sections compared with controls and thus confirmed the results obtained at the mRNA level. In vitro data derived from PASMCs further corroborated the observed downregulation of
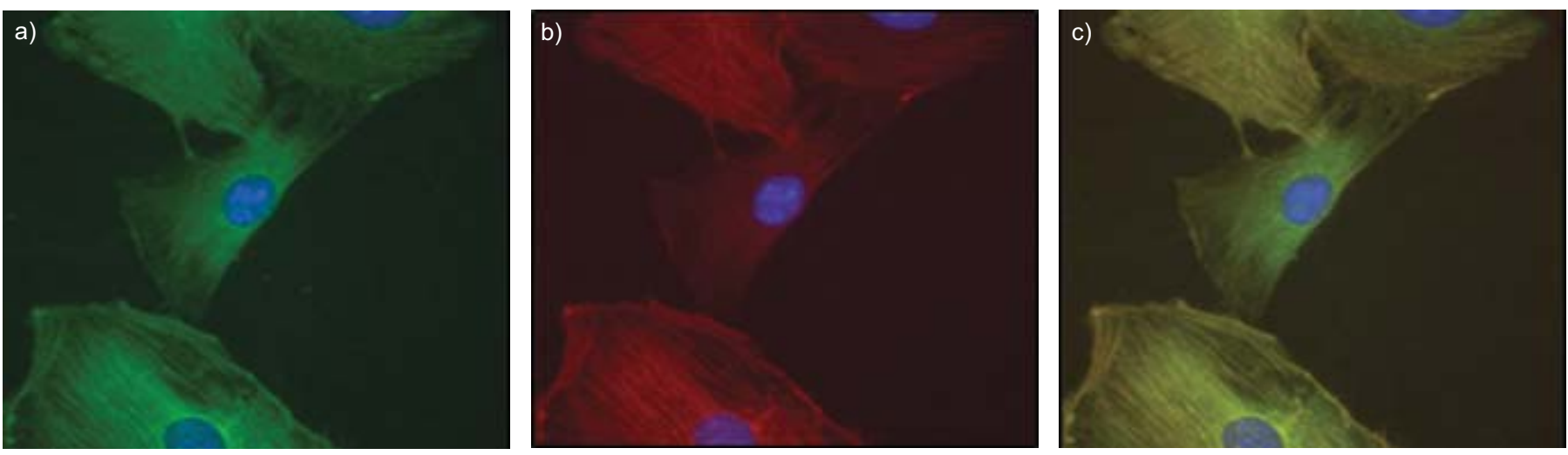

FIGURE 6. Immunofluorescence analysis of primary mouse primary arterial smooth muscle cells (PASMCs) demonstrates a cytoplasmic pattern for a) Shroom and b) $\alpha$-smooth-muscle actin. c) A merged image demonstrating co-localisation of both proteins in PASMCs. 

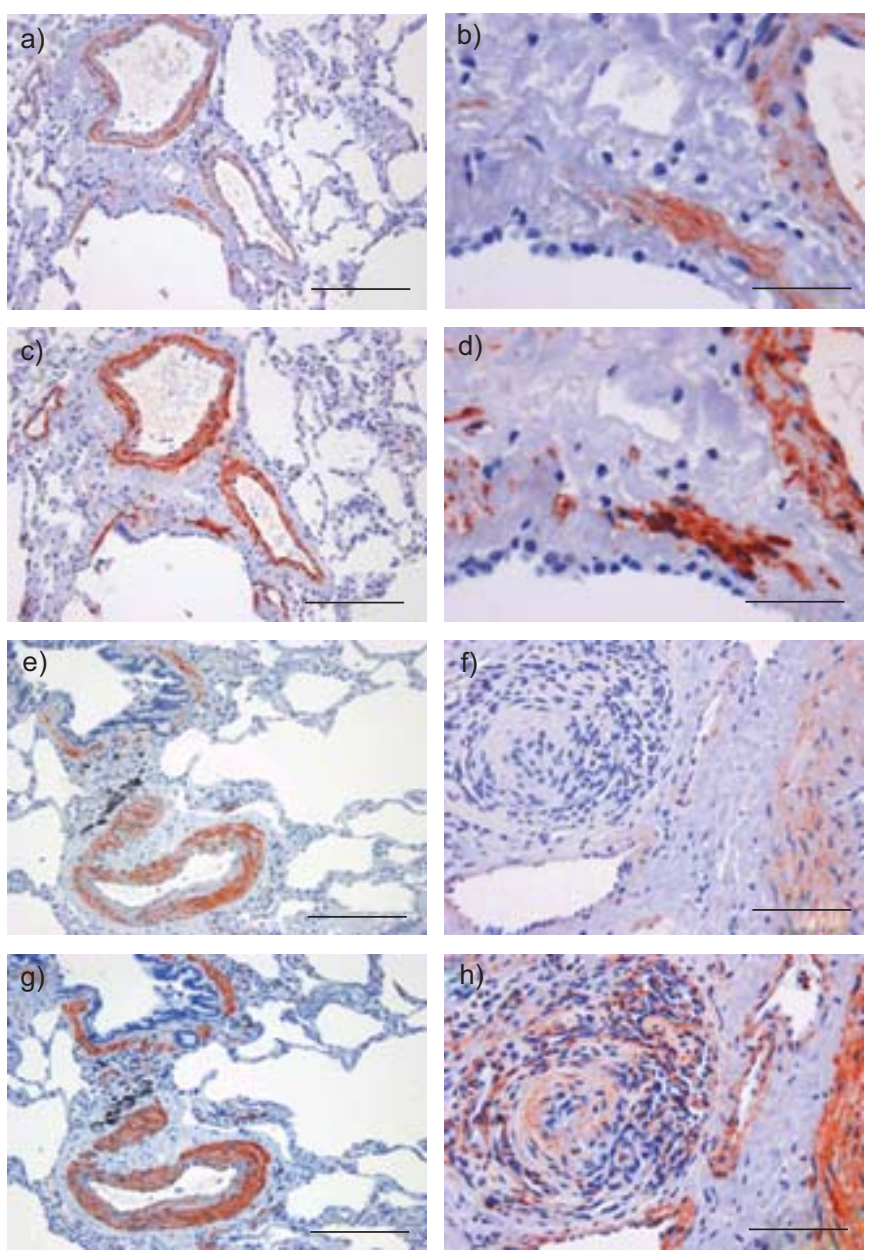

FIGURE 7. Shroom protein localisation in the human lung. Representative serial immunohistochemical sections illustrate a similar spatial expression of Shroom ( $a, b$, e and f) and $\alpha$-smooth-muscle actin (c, d, g and h) in smooth muscle cells, both in low ( $a, c, e$ and $g$ ) and high ( $b, d, f$ and $h$ ) magnification. $a, b, c$ and d) Staining of normal human lungs (transplant donors) and $\mathrm{e}, \mathrm{f}, \mathrm{g}$ and $\mathrm{h}$ ) staining of idiopathic pulmonary arterial hypertension lungs. $f$ and $h$ ) Stainings in and around a plexiform lesion derived from an IPAH patient with no detectable bmpr2 mutations. a, c, e and g) Scale bars $=100 \mu \mathrm{m}$; b and d) scale bars $=50 \mu \mathrm{m}$; $f$ and $h$ ) scale bars $=100 \mu \mathrm{m}$.

shrm expression by hypoxia, together suggesting that Shroom expression was attenuated during the development of $\mathrm{PAH}$. Given that PASMC de-differentiation and subsequent activation is a key pathophysiological parameter during the development $\mathrm{PAH}$, it is reasonable to speculate that persistent Shroom expression may be required for maintenance of the quiescent PASMC phenotype. It is presently unclear, however, whether attenuation of Shroom expression is an epiphenomenon, or is the cause of PASMC de-differentiation and activation.

The shroom gene family currently consists of four members: shroom1 (also called APXL2 or KIAA1960), shroom2 (also called APXL, DKFZp781J074, FLJ39277 or HSAPXL), shroom3 (formerly called Shroom, but also called APXL3, KIAA1481 or MSTP013) and shroom4 (also called RP11-119E20.1, KIAA1202), in line with a recently proposed new nomenclature [21] and the updated human genome resources database at the National
Center for Biotechnology Information [22]. These four members can be distinguished by several structural differences: while the PDZ and ASD1 domains are present in all members of the family, the ASD2 domain is absent in Shroom4 [12, 23]. Detailed future investigations into the spatiotemporal, but also species-specific expression pattern of all Shroom family members are required to fully investigate their role in PASMC hypertrophy in disease, as suggested herein. Of all these members, only Shroom 4 has been described before to be expressed in the murine and human lung [23,24]. Thus far, the other Shroom members have been localised in neural epithelial cells [12] and fibroblasts [13].

In light of these reports, the intense Shroom staining in murine and human lung PASMC was surprising, as it has not been previously described. What could be the possible functions of Shroom in PASMC? Considering that Shroom is an actinbinding protein, which directly regulates reorganisation of the actin cytoskeleton upon cell de-differentiation and/or transformation, it is reasonable to assume that Shroom controls the actin cytoskeleton in PASMCs. PASMCs are the primary cell type that expresses $\alpha$-SMA in the lung, along with myofibroblasts under disease conditions, such as pulmonary fibrosis. Therefore, Shroom may control the cytoskeleton of the quiescent PASMCs under normal conditions, a regulatory function that is lost upon decreased expression of Shroom in vascular disorders such as $\mathrm{PAH}$.

A large number of animal models have been utilised to study the pathogenesis of $\mathrm{PAH}$, including the mouse model of chronic hypoxia-induced $\mathrm{PAH}$, where muscularisation occurs in distal arterioles, with subsequent increases in right ventricular mass and pressure [25]. These processes are preceded by select alterations in the gene expression profile of cells within the vascular wall, in particular PASMCs and adventitial fibroblasts $[6,26]$. Particularly relevant to hypoxiainduced effects are the regulatory HRE regions in the promoter and enhancer sequences of hypoxia-regulated genes. While a large number of reports have thus far demonstrated that increased HIF-1 activity can lead to increased gene transcription, only a few reports have reported the inhibition of gene transcription via HRE sites [27, 28]. As such, the four putative HRE sites found in the murine shrm promoter and enhancer may provide important clues for understanding of HIFmediated inhibition of gene transcription in response to hypoxia. In this respect, future investigations deciphering the functional contribution of sequences that are immediately adjacent to these HRE sites are required to delineate any possible regulatory sequences mediating inhibition of gene transcription in the context of hypoxia.

Major agents promote PASMC migration, such as endothelin-1 and chemokines, including chemokine (C-C motif) ligand 2 (monocyte chemotactic protein-1) and chemokine (C-X3-C motif) ligand 1 (fractalkine) [29]. These ligands induce actin polymerisation in the cell, when bound to the corresponding chemokine receptors, and thus influence cell migration. Further study on the influence of these ligands on Shroom localisation and expression may highlight a possible therapeutic benefit of targeting Shroom or other cytoskeletal proteins in the context of IPAH. The data in the present study also demonstrate that Shroom is expressed in airway PASMC, as 

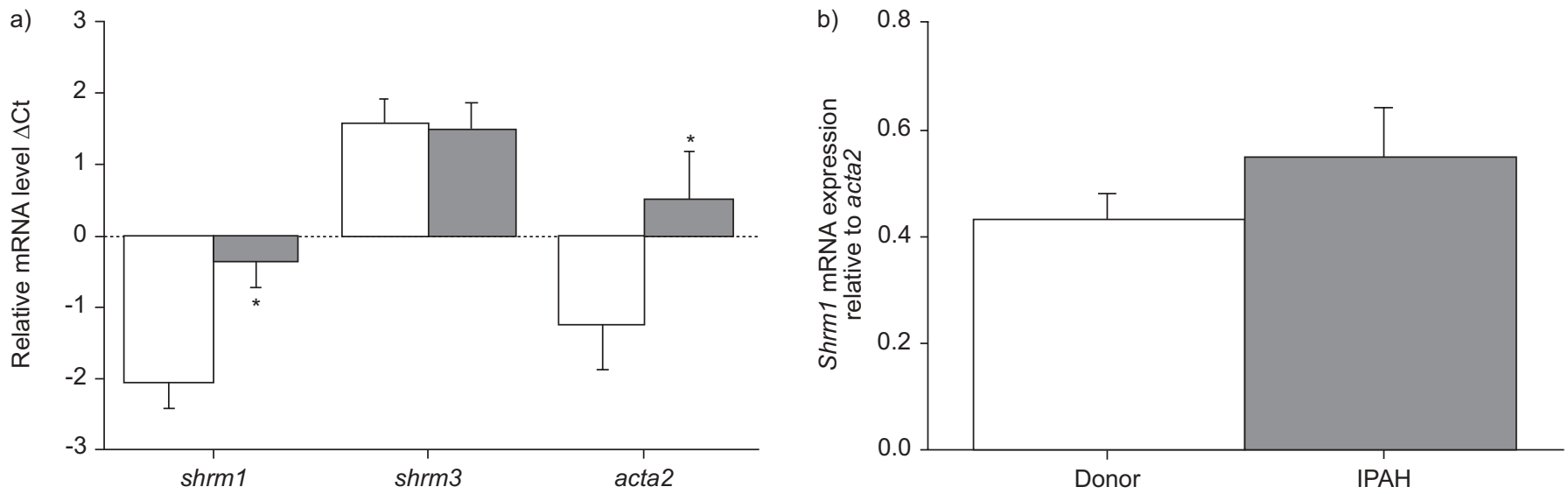

FIGURE 8. Gene expression in the human lung. a) Expression levels of shrm1, shrm3 or $\alpha$-smooth muscle cell actin (acta2) in lungs from healthy human donors ( $\square$ ) or from idiopathic pulmonary arterial hypertension (IPAH) patients ( $\square$ ) are presented. b) The expression ratio of shrm/acta2 in control and diseased lungs is represented calculated from the data presented in a); fold change $=2^{\Delta \Delta C t}$. Data are presented as mean \pm SEM. $\Delta C t$ and $\Delta \Delta C$ t are defined in Materials and Methods: RT-PCR. *: $p<0.05$.

well as in vascular smooth muscle cells. This observation may be relevant to diseases that are characterised by airway smooth muscle hypertrophy, such as bronchial asthma [30], in which Shroom, by virtue of its regulation of smooth muscle homeostasis, may also play a pathogenic role.

In both whole-lung homogenates and cultured human PASMCs from patients with IPAH, it is noteworthy that the effects of standard therapies for PAH (endothelin receptor antagonists, type- 5 phosphodiesterase inhibitors and prostacyclin derivatives) on Shroom expression have not been fully investigated due to the variety of treatments available. Therefore, it remains possible that pharmacological interventions in the patient population may also have influenced shrm RNA levels assessed in the present study. the apparent discrepancy of the results documenting shrm expression levels in whole lung homogenates from mouse and human samples was initially surprising. While shrm expression was decreased in mouse lungs of chronic hypoxia-induced PAH (fig. 2a), this difference was not observed in human lungs from IPAH patients (fig. 7a). This could be explained as follows. First, the nomenclature of the shrm genes has recently evolved and novel family members are still being discovered. A close comparison of mouse and human shrm genes may therefore unravel novel homologues in the future, which then warrant detailed investigations in diseases associated with PASMC hypertrophy. Secondly, as the IPAH patients investigated herein presented with late-stage $\mathrm{PAH}$, compensatory mechanisms in the human disease may have accounted for reactive changes in shrm expression, which were not operative in the short-term mouse model.

In conclusion, the present study reports a novel protein, Shroom, which is possibly involved in PAH pathogenesis. While recent data has implied that the bone BMPR2 and activin-like kinase-1 in familial cases of PAH [31, 32], as well as angiopoietin-1, Tie-2 and serotonin in all forms of PAH [33], the precise molecular mechanisms that lead to the evolution of PAH still remain elusive. Despite this, the PASMC has emerged as a key cell-type that causally contributes to the
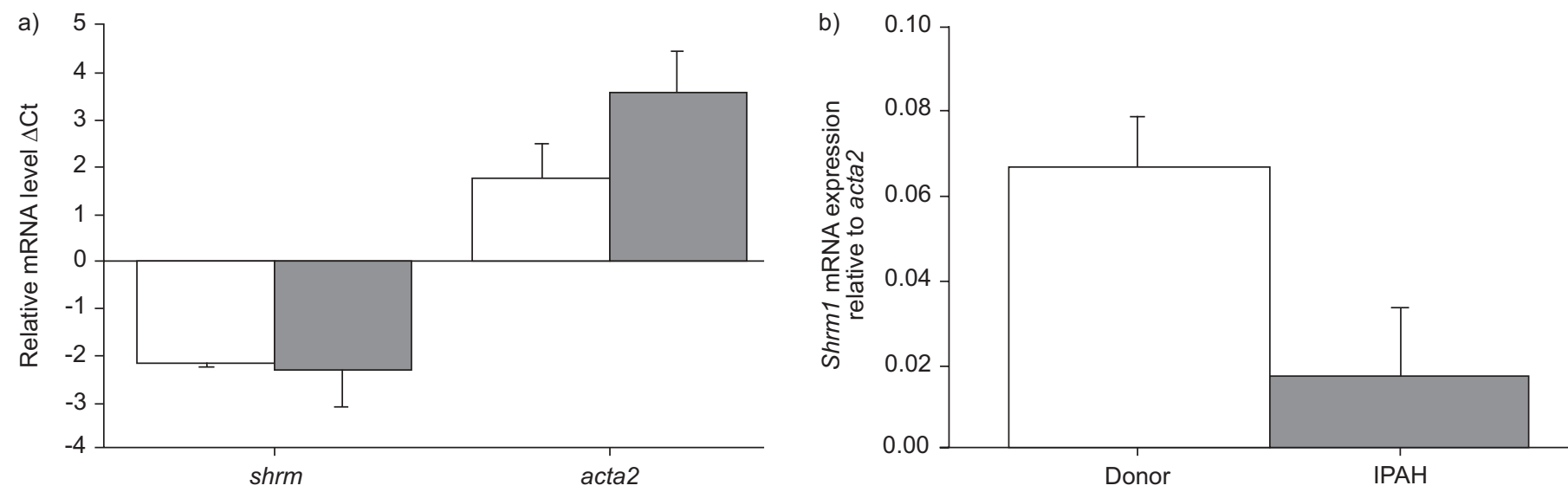

FIGURE 9. Gene expression in primary pulmonary arterial smooth muscle cells cultured from donor or idiopathic pulmonary arterial hypertension lungs. a) Expression levels of shrm1 and $\alpha$-smooth muscle cell actin (acta2) in pulmonary artery smooth muscle cells cultured from the lungs from healthy human donors ( $\square$ ) and from idiopathic pulmonary arterial hypertension (IPAH) patients $(\square)$ are presented. b) The ratios between shrm/acta2 in control and diseased lungs were derived from the data presented in a); fold change $=2^{\Delta \Delta C t}$. Data are presented as mean \pm SEM. $\Delta \mathrm{Ct}$ and $\Delta \Delta \mathrm{Ct}$ are defined in Materials and Methods: RT-PCR. 
increased vascular resistance in PAH. As such, the analysis of PASMC hypertrophy under disease conditions represents a valuable tool in investigating novel approaches to disease pathogenesis. Since the maintenance of cytoskeletal actin is crucial for cell shape, intracellular trafficking of compartmentalised cellular components and PASMC contraction, and thus quiescence [7], the present authors postulate that alteration to the actin organisation, possibly due to the absence of Shroom, leads to uncontrolled PASMC hypertrophy and finally, the development of PAH.

Clearly, selective and specific modification of pulmonary arterial smooth-muscle cell hypertrophy in pulmonary arterial hypertension may present as a valuable therapeutic tool in future investigations.

\section{ACKNOWLEDGEMENTS}

We are indebted to M.S. Krishnaveni (Division of Pulmonary and Critical Care Medicine, University of Southern California, Los Angeles, CA, USA) for critical reading of this manuscript and the Graduate Programme "Molecular Biology and Medicine of the Lung" class of 2007 for invaluable discussions.

\section{REFERENCES}

1 Gaine SP, Rubin LJ. Primary pulmonary hypertension. Lancet 1998; 352: 719-725.

2 Humbert M, Sitbon O, Simonneau G. Treatment of pulmonary arterial hypertension. N Engl J Med 2004; 351: 1425-1436.

3 Runo JR, Loyd JE. Primary pulmonary hypertension. Lancet 2003; 361: 1533-1544.

4 Humbert M, Morrell NW, Archer SL, et al. Cellular and molecular pathobiology of pulmonary arterial hypertension. J Am Coll Cardiol 2004; 43: Suppl. 12, 13S-24S.

5 Eickelberg O, Yeager ME, Grimminger F. The tantalizing triplet of pulmonary hypertension-BMP receptors, serotonin receptors, and angiopoietins. Cardiovasc Res 2003; 60: 465-467.

6 Stenmark KR, Fagan KA, Frid MG. Hypoxia-induced pulmonary vascular remodeling: cellular and molecular mechanisms. Circ Res 2006; 99: 675-691.

7 Solway J, Forsythe SM, Halayko AJ, Vieira JE, Hershenson MB, Camoretti-Mercado B. Transcriptional regulation of smooth muscle contractile apparatus expression. Am J Respir Crit Care Med 1998; 158: S100-S108.

8 Day RM, Agyeman AS, Segel MJ, et al. Serotonin induces pulmonary artery smooth muscle cell migration. Biochem Pharmacol 2006; 71: 386-397.

9 Schmidt A, Hall MN. Signaling to the actin cytoskeleton. Annu Rev Cell Dev Biol 1998; 14: 305-338.

10 Palmgren S, Vartiainen M, Lappalainen P. Twinfilin, a molecular mailman for actin monomers. J Cell Sci 2002; 115: 881-886.

11 Pollard TD, Blanchoin L, Mullins RD. Molecular mechanisms controlling actin filament dynamics in nonmuscle cells. Annu Rev Biophys Biomol Struct 2000; 29: 545-576.

12 Hildebrand JD, Soriano P. Shroom, a PDZ domaincontaining actin-binding protein, is required for neural tube morphogenesis in mice. Cell 1999; 99: 485-497.
13 Dietz ML, Bernaciak TM, Vendetti F, Kielec JM, Hildebrand JD. Differential actin-dependent localization modulates the evolutionarily conserved activity of Shroom family proteins. J Biol Chem 2006; 281: 20542-20554.

14 Morty RE, Nejman B, Kwapiszewska G, et al. Dysregulated bone morphogenetic protein signaling in monocrotalineinduced pulmonary arterial hypertension. Arterioscler Thromb Vasc Biol 2007; 27: 1072-1078.

15 Zakrzewicz A, Kouri FM, Nejman B, et al. The TGF- $\beta$ / Smad2,3 signalling axis is impaired in experimental pulmonary hypertension. Eur Respir J 2007; 29: 1094-1104.

16 Dumitrascu R, Weissmann N, Ghofrani HA, et al. Activation of soluble guanylate cyclase reverses experimental pulmonary hypertension and vascular remodeling. Circulation 2006; 113: 286-295.

17 Fink L, Seeger W, Ermert L, et al. Real-time quantitative RT-PCR after laser-assisted cell picking. Nat Med 1998; 4 1329-1333.

18 Alejandre-Alcázar MA, Kwapiszewska G, Reiss I, et al. Hyperoxia modulates TGF- $\beta$ /BMP signaling in a mouse model of bronchopulmonary dysplasia. Am J Physiol Lung Cell Mol Physiol 2007; 292: L537-L549.

19 Semenza GL. Regulation of physiological responses to continuous and intermittent hypoxia by hypoxia-inducible factor 1. Exp Physiol 2006; 91: 803-806.

20 Kwapiszewska G, Wilhelm J, Wolff S, et al. Expression profiling of laser-microdissected intrapulmonary arteries in hypoxia-induced pulmonary hypertension. Respir Res 2005; 6: 109.

21 Hagens O, Ballabio A, Kalscheuer V, et al. A new standard nomenclature for proteins related to Apx and Shroom. BMC Cell Biol 2006; 7: 18.

22 National Center for Biotechnology Information. Human Genomes Resources. www.ncbi.nlm.nih.gov/projects / genome/guide/human Date last accessed and updated: April 13, 2007.

23 Yoder M, Hildebrand JD. Shroom4 (Kiaa1202) is an actinassociated protein implicated in cytoskeletal organization. Cell Motil Cytoskeleton 2007; 64: 49-63.

24 Hagens O, Dubos A, Abidi F, et al. Disruptions of the novel KIAA1202 gene are associated with X-linked mental retardation. Hum Genet 2006; 118: 578-590.

25 Weissmann N, Akkayagil E, Quanz K, et al. Basic features of hypoxic pulmonary vasoconstriction in mice. Respir Physiol Neurobiol 2004; 139: 191-202.

26 Stenmark KR, Frid M, Nemenoff R, Dempsey EC, Das M. Hypoxia induces cell-specific changes in gene expression in vascular wall cells: implications for pulmonary hypertension. Adv Exp Med Biol 1999; 474: 231-258.

27 Erler JT, Cawthorne CJ, Williams KJ, et al. Hypoxiamediated down-regulation of Bid and Bax in tumors occurs via hypoxia-inducible factor 1-dependent and -independent mechanisms and contributes to drug resistance. Mol Cell Biol 2004; 24: 2875-2889.

28 Narravula S, Colgan SP. Hypoxia-inducible factor 1mediated inhibition of peroxisome proliferator-activated receptor $\alpha$ expression during hypoxia. J Immunol 2001; 166: 7543-7548.

29 Perros F, Dorfmüller P, Souza R, et al. Fractalkine-induced smooth muscle cell proliferation in pulmonary hypertension. Eur Respir J 2007; 29: 937-943. 
30 Bentley JK, Hershenson MB. Airway smooth muscle growth in asthma: proliferation, hypertrophy, and migration. Proc Am Thorac Soc 2008; 5: 89-96.

31 Machado RD, Aldred MA, James V, et al. Mutations of the TGF- $\beta$ type II receptor BMPR2 in pulmonary arterial hypertension. Hum Mutat 2006; 27: 121-132.
32 Morrell NW. Pulmonary hypertension due to BMPR2 mutation: a new paradigm for tissue remodeling? Proc Am Thorac Soc 2006; 3: 680-686.

33 Sullivan CC, Du L, Chu D, et al. Induction of pulmonary hypertension by an angiopoietin 1/TIE2/serotonin pathway. Proc Natl Acad Sci USA 2003; 100: 12331-12336. 